\title{
Erratum to: Impact of the expression of thymidylate synthase and dihydropyrimidine dehydrogenase genes on survival in stage II/III gastric cancer
}

\author{
Mitsuru Sasako - Masanori Terashima - Wataru Ichikawa - Atsushi Ochiai • \\ Koji Kitada • Issei Kurahashi - Shinichi Sakuramoto - Hitoshi Katai • \\ Takeshi Sano $\cdot$ Hiroshi Imamura
}

Published online: 8 October 2014

(C) The Author(s) 2014. This article is published with open access at Springerlink.com

\section{Erratum to: Gastric Cancer \\ DOI 10.1007/s10120-014-0413-8}

Unfortunately, errors appeared in this article.

In the Results section, under the heading "Predictive value of biomarker analysis", at the end of the final sentence in the second paragraph, the parenthetical expression "(data not shown)" should be deleted, as the data actually are shown.
Also, in Table 4, for the factor "Histological type" (in column 1), in column 2 ("Group") the word "Undifferentiated" should appear first, above "Differentiated", and the superscript "a" on "Undifferentiated" should be deleted.

We apologize for the occurrence of these errors.

Open Access This article is distributed under the terms of the Creative Commons Attribution License which permits any use, distribution, and reproduction in any medium, provided the original author(s) and the source are credited.

\footnotetext{
I. Kurahashi

Department of Medical Informatics and Economics,

The University of Tokyo, 7-3-1 Hongo, Bunkyo,

Tokyo 113-0033, Japan

\section{S. Sakuramoto}

Department of Surgery, Saitama Medical University, International Medical Center, 1397-1 Yamane, Hidaka, Saitama 350-1298, Japan

H. Katai

Gastric Surgery Division, National Cancer Center Hospital, 5-1-1 Tsukiji, Chuo, Tokyo 104-0045, Japan

T. Sano

Department of Gastroenterological Surgery, Cancer Institute Hospital, Japanese Foundation for Cancer Research, 3-8-31 Ariake, Koto, Tokyo 135-8550, Japan

\section{H. Imamura}

Department of Surgery, Toyonaka Municipal Hospital,

4-14-1 Shibahara-cho, Toyonaka, Osaka 560-8565, Japan
} 